\title{
Pore Scale Study of Dynamic Adsorption Process in Capacitive deionization Porous Electrode Based on Lattice Boltzmann Method
}

\author{
Shouguang Yao*, Xi Ding, Yi Ji, Xin Kan, Rui Liu \\ School of Energy and Power Engineering, Jiangsu University of Science and Technology, 212003, \\ Zhenjiang, Jiangsu, China \\ *E-mail: zjyaosg@126.com
}

doi: $10.20964 / 2021.08 .16$

Received: 2 April 2021 / Accepted: 26 May 2021 / Published: 30 June 2021

Capacitive deionization (CDI) has become a promising desalination technology due to its low energy consumption and environmental friendliness. Understanding the transport mechanism of ions within CDI porous electrodes is essential to improve desalination performance. In this study, a numerical model coupled with the Navier-Stokes equations, the concentration-diffusion equation, and the Langmuir isothermal adsorption equation is developed for the first time to describe the transport process in a porous electrode. To facilitate the calculations, a simplified particle model is used to describe the distribution of the particles in the porous electrode. With the discrete solution of the numerical model by means of the Lattice Boltzmann method, the transient changes of $\mathrm{Na}, \mathrm{Cl}$ ion concentrations in the solution and the particles are accurately described. Using this numerical model, the effects of inlet solution velocity, electrode porosity and porous skeleton particle size on the internal mass transfer and adsorption performance of the CDI porous electrode were investigated at the pore scale. The results show that an appropriate increase in the inlet solution flow rate can effectively improve the adsorption amount and rate of the porous electrode. The increase of porosity improves the utilization rate and the average adsorption capacity of the electrode material. Decreasing the particle size of porous skeleton particles can reduce the internal mass transfer resistance of the porous electrode and enhance the adsorption performance of the electrode. This study shows that pore-scale simulations can help to understand the ion transport and adsorption mechanisms in capacitive deionization electrode particles and point the way for the next optimization.

Keywords: Capacitive deionization, Porous electrode, Langmuir, Lattice Boltzmann method, Porescale

\section{FULL TEXT}


(C) 2021 The Authors. Published by ESG (www.electrochemsci.org). This article is an open access article distributed under the terms and conditions of the Creative Commons Attribution license (http://creativecommons.org/licenses/by/4.0/). 